\title{
Observing crosswind over urban terrain using scintillometer and Doppler lidar
}

\author{
D. van Dinther ${ }^{1}$, C. R. Wood ${ }^{2}$, O. K. Hartogensis ${ }^{1}$, A. Nordbo ${ }^{3}$, and E. J. O'Connor ${ }^{2,4}$ \\ ${ }^{1}$ Meteorology and Air Quality Group, Wageningen University, Wageningen, the Netherlands \\ ${ }^{2}$ Finnish Meteorological Institute, Helsinki, Finland \\ ${ }^{3}$ Department of Physics, University of Helsinki, Helsinki, Finland \\ ${ }^{4}$ Department of Meteorology, University of Reading, Reading, UK
}

Correspondence to: C. R. Wood (curtis.wood@fmi.fi)

Received: 28 April 2014 - Published in Atmos. Meas. Tech. Discuss.: 1 July 2014

Revised: 26 March 2015 - Accepted: 6 April 2015 - Published: 24 April 2015

\begin{abstract}
In this study, the crosswind (wind component perpendicular to a path, $U_{\perp}$ ) is measured by a scintillometer and estimated with Doppler lidar above the urban environment of Helsinki, Finland, for 15 days. The scintillometer allows acquisition of a path-averaged value of $U_{\perp}\left(\overline{U_{\perp}}\right)$, while the lidar allows acquisition of path-resolved $U_{\perp}\left(U_{\perp}(x)\right.$, where $x$ is the position along the path). The goal of this study is to evaluate the performance of scintillometer $\overline{U_{\perp}}$ estimates for conditions under which $U_{\perp}(x)$ is variable. Two methods are applied to estimate $\overline{U_{\perp}}$ from the scintillometer signal: the cumulative-spectrum method (relies on scintillation spectra) and the look-up-table method (relies on time-lagged correlation functions). The values of $\overline{U_{\perp}}$ of both methods compare well with the lidar estimates, with root-mean-square deviations of 0.71 and $0.73 \mathrm{~m} \mathrm{~s}^{-1}$. This indicates that, given the data treatment applied in this study, both measurement technologies are able to obtain estimates of $\overline{U_{\perp}}$ in the complex urban environment. The detailed investigation of four cases indicates that the cumulative-spectrum method is less susceptible to a variable $U_{\perp}(x)$ than the look-up-table method. However, the look-up-table method can be adjusted to improve its capabilities for estimating $\overline{U_{\perp}}$ under conditions under for which $U_{\perp}(x)$ is variable.
\end{abstract}

\section{Introduction}

The general application of a scintillometer in micrometeorology is obtaining path-averaged surface fluxes (among others De Bruin, 2002; Meijninger et al., 2002a, b). The path can range from a few hundred metres to a few kilometres depending on the type of scintillometer used (De Bruin, 2002). In this study, the focus is on obtaining the path-averaged crosswind from a scintillometer (among others Briggs et al., 1950; Wang et al., 1981), where the crosswind $\left(U_{\perp}\right)$ is defined as the wind component perpendicular to the scintillometer path. By obtaining a path-averaged value of $U_{\perp}\left(\overline{U_{\perp}}\right)$ instead of a point measurement, a scintillometer is more suitable to validate wind fields calculated by models - given the resolution of numerical weather prediction models $(\sim 10 \mathrm{~km})$. Furthermore, point measurements can more easily be biased than path-averaged values, especially for urban areas at heights within about 2-3 times the canopy-layer depth (the canopy layer is typically defined as the average building height).

From scintillometer data, one can obtain $\overline{U_{\perp}}$ from either the scintillation power spectrum $\left(S_{11}(f)\right.$, where $f$ is the frequency) (van Dinther et al., 2013) or the time-lagged correlation function $\left(r_{12}(\tau)\right.$, where $\tau$ is the time lag) (among others Briggs et al., 1950; Poggio et al., 2000; van Dinther and Hartogensis, 2014). Validation attempts of $\overline{U_{\perp}}$ have mainly taken place at flat grassland sites (Poggio et al., 2000; van Dinther et al., 2013). At such sites, $U_{\perp}$ is assumed to be uniform along the scintillometer path. Furthermore, there is also a need for scintillometer $\overline{U_{\perp}}$ in more complex areas, such as mountain environments (Poggio et al., 2000) and urban environments (above the River Thames in London in Wood et al., 2013c). Ward et al. (2011) studied the influence of a variable $U_{\perp}$ field along the path $\left(U_{\perp}(x)\right.$, where $x$ is the location on the scintillometer path) on the scintillometer signal however, their focus was on scintillation spectra and structure 
parameter estimates rather than on $\overline{U_{\perp}}$ estimates. The $U_{\perp}(x)$ fields used in their study were synthetic. In the present study, the focus is on the influence of a measured variable $U_{\perp}(x)$ on the $\overline{U_{\perp}}$ estimate of a scintillometer.

The measurements investigated in this study are taken in the urban environment. In such an environment, the wind speed and direction are spatially variable (Bornstein and Johnson, 1977), making it a suitable environment to study the influence of a variable $U_{\perp}(x)$ on the scintillometer estimates of $\overline{U_{\perp}}$. Key to this study are measurements of the variability of $U_{\perp}(x)$ that are estimated by a scanning Doppler lidar. In this experiment the lidar was configured in a horizontal scan pattern, in order to estimate the horizontal wind speed and wind direction along the scintillometer path using a duo-beam method (Wood et al., 2013c).

The measurements were taken in Helsinki, Finland, as part of the Helsinki Urban Boundary-layer Atmosphere Network (Helsinki UrBAN; Wood et al., 2013a, http://urban.fmi.fi). The spatial and temporal variability of $U_{\perp}(x)$ induced by buildings poses challenges for both the lidar and the scintillometer technologies: (i) the lidar, since one assumes homogeneity of the wind field within each range gate (sampling bin) for both beams in the lidar duo-beam pair, and (ii) the scintillometer, since both $S_{11}(f)$ and $r_{12}(\tau)$ used in the $\overline{U_{\perp}}$ retrieval algorithms are influenced by a variable $U_{\perp}(x)$, although the algorithms do not take this into account (van Dinther et al., 2013; van Dinther and Hartogensis, 2014). We are, therefore, working at the limit of both measurement technologies.

The main goal of this study is to investigate the performance of the scintillometer to measure $\overline{U_{\perp}}$ in conditions for which $U_{\perp}(x)$ is variable. In order to do so, estimates from the scintillometer of $\overline{U_{\perp}}$ are compared to estimates from the lidar. However, also for the lidar, heterogeneous wind conditions are challenging. Therefore, before the scintillometer and lidar $\overline{U_{\perp}}$ estimates are compared to each other, the applicability of the lidar to estimate $U_{\perp}(x)$ is investigated by comparing with sonic-anemometer measurements. Lastly, four cases will be selected where lidar-estimated $U_{\perp}(x)$ values are used to obtain the theoretical $S_{11}(f)$ and $r_{12}(\tau)$, from the models given by Clifford (1971) and Lawrence et al. (1972), respectively. The influence of a variable $U_{\perp}(x)$ on the theoretical $S_{11}(f)$ and $r_{12}(\tau)$ gives insight into the robustness of the scintillometer methods to obtain $\overline{U_{\perp}}$.

\section{Theory and methods}

\subsection{Scintillometry}

A scintillometer comprises a transmitter and a receiver. Here, a large-aperture scintillometer is used and its transmitter emits near-infrared radiation, which is scattered by constantly changing eddies as it passes through the turbulent atmosphere. Hence, the intensity measured by the receiver fluctuates on short timescales $(\sim 1 \mathrm{~s})$. For these timescales Taylor's frozen-turbulence assumption is valid, making $U_{\perp}$ the only driver of changes in the eddy field.

The value of $\overline{U_{\perp}}$ can be obtained from the intensity fluctuations (also referred to as scintillation signal) by either the scintillation power spectrum or time-lagged correlation function. The benefit of the methods relying on $r_{12}(\tau)$ instead of $S_{11}(f)$ is that also the crosswind direction (i.e. the sign of $\overline{U_{\perp}}$ ) can be obtained from $r_{12}(\tau)$. Another benefit is that $r_{12}(\tau)$ can be determined over a short timescale $(\sim 10 \mathrm{~s})$, while $S_{11}(f)$ needs to be determined over a longer timescale $(\sim 10 \mathrm{~min})$. On the other hand, $r_{12}(\tau)$ needs to be obtained from a dual-aperture scintillometer, while scintillation spectra be obtained from every type of scintillometer.

In this study we use the cumulative-spectrum method to obtain $\overline{U_{\perp}}$ from $S_{11}(f)$ (van Dinther et al., 2013) and the look-up-table method to obtain $\overline{U_{\perp}}$ from $r_{12}(\tau)$ (van Dinther and Hartogensis, 2014). A detailed description of the methods is given in van Dinther et al. (2013) and van Dinther and Hartogensis (2014); a brief outline of the methods is given below.

\subsubsection{Scintillation spectra}

The scintillation spectrum $\left(S_{11}(f)\right)$ gives insight into which frequencies contribute to the variance of the scintillation signal. Clifford (1971) describes a theoretical model of the scintillation spectrum. Adjusting this model for the largeaperture scintillometer gives (Nieveen et al., 1998)

$$
\begin{aligned}
& S_{11}(f)=16 \pi^{2} k^{2} \int_{0}^{1} \int_{2 \pi f / U_{\perp}(x)}^{\infty} \\
& K \phi_{n}(K) \sin ^{2}\left(\frac{K^{2} L x(1-x)}{2 k}\right)\left[\left(K U_{\perp}(x)\right)^{2}-(2 \pi f)^{2}\right]^{-1 / 2} \\
& \left(\frac{2 J_{1}\left(0.5 K D_{\mathrm{R}} x\right)}{0.5 K D_{\mathrm{R}} x}\right)^{2}\left(\frac{2 J_{1}\left(0.5 K D_{\mathrm{T}}(1-x)\right)}{0.5 K D_{\mathrm{T}}(1-x)}\right)^{2} \mathrm{~d} K \mathrm{~d} x,
\end{aligned}
$$

where $f$ is the frequency at which $S_{11}$ is representative, $k$ is the wavenumber of the emitted radiation, $K$ the turbulent spatial wave number, $L$ is the scintillometer path length, $x$ is the relative location on the path, $J_{1}$ is the first-order Bessel function of the first kind, $D_{\mathrm{R}}$ is the aperture diameter of the receiver, $D_{\mathrm{T}}$ is the aperture diameter of the transmitter, and $\phi_{n}(K)$ is the three-dimensional spectrum of the refractive index in the inertial range given by Kolmogorov (1941). As can be seen in Eq. (1), $U_{\perp}(x)$ influences the scintillation spectrum. In fact, the scintillation spectrum shifts linearly across the frequency axis as a function of $\overline{U_{\perp}}$. Therefore, by obtaining a characteristic point in the spectrum, $\overline{U_{\perp}}$ can be obtained (van Dinther et al., 2013).

The cumulative spectrum is obtained by integrating a scintillation spectrum from low to high frequency and normalizing this integration by the variance in the scintillation signal. The cumulative-spectrum method takes into account multiple 
characteristic frequency points $\left(f_{\mathrm{CS}}\right)$, which here are defined as the frequency points for which the cumulative spectrum equals $0.5,0.6,0.7,0.8$, and 0.9 (as in van Dinther et al., 2013). For each of these five points, a value of $\overline{U_{\perp}}$ is determined by

$\overline{U_{\perp}}=C_{\mathrm{CS}} \cdot f_{\mathrm{CS}}$,

where $C_{\mathrm{CS}}$ is a unique constant which depends on the experimental setup and scintillometer used. Derivation of $C_{\mathrm{CS}}$ is possible from the theoretical $S_{11}(f)$ (Eq. 1) by filling in values of $U_{\perp}$ and assuming that $U_{\perp}(x)$ is constant for the five different frequency points. Subsequently, the five different $\overline{U_{\perp}}$ values are averaged to obtain one value of $\overline{U_{\perp}}$ per cumulative spectrum. In this study, we will investigate to what extent the assumption that $C_{\mathrm{CS}}$ is constant holds when $U_{\perp}(x)$ varies. This investigation is carried out by means of four cases for which the $U_{\perp}(x)$ estimates of the lidar are used in Eq. (1) to obtain the theoretical $S_{11}(f)$. Therefore, Eq. (1) is not integrated for $x$ over 0 to 1 , but over the 136 range gates measured by the lidar (see Sect. 4.3). In this study, cumulative spectra are obtained over periods of $10 \mathrm{~min}$.

\subsubsection{Time-lagged correlation function}

The value of $\overline{U_{\perp}}$ can be obtained from a dual-aperture scintillometer (scintillometer with horizontally displaced beams) using $r_{12}(\tau)$. For a dual-aperture scintillometer, the two transmitters and receivers are in general displaced by only a small distance $(\sim 10 \mathrm{~cm})$. This small spatial difference means that the eddy field barely changes as the wind transports it through one beam to the next (i.e. the frozenturbulence assumption is not unreasonable). The signals of the two spatially separated scintillometer beams should thus be almost identical except for a time shift. This time shift is related to $\overline{U_{\perp}}$, and can be obtained from $r_{12}(\tau)$. A theoretical model of the time-lagged covariance function $\left(C_{12}(\tau)\right)$ is given by Lawrence et al. (1972), here including the largeaperture averaging terms of Wang et al. (1978):

$$
\begin{aligned}
& C_{12}(\tau)=16 \pi^{2} k^{2} \int_{0}^{1} \int_{0}^{\infty} \\
& K \phi_{n}(K) \sin ^{2}\left[\frac{K^{2} L x(1-x)}{2 k}\right] J_{0}\left\{K\left[s(x)-U_{\perp}(x) \tau\right]\right\} \\
& {\left[\frac{2 J_{1}\left(0.5 K D_{\mathrm{R}} x\right)}{0.5 K D_{\mathrm{R}} x}\right]^{2}\left\{\frac{2 J_{1}\left[0.5 K D_{\mathrm{T}}(1-x)\right]}{0.5 K D_{\mathrm{T}}(1-x)}\right\}^{2} \mathrm{~d} K \mathrm{~d} x,}
\end{aligned}
$$

where $J_{0}$ is the zero-order Bessel function of the first kind, and $s(x)$ is the separation distance between the two beams at location $x$. The theoretical $r_{12}(\tau)$ can be obtained by dividing the theoretical $C_{12}(\tau)$ by the theoretical $C_{11}(\tau)$, where $C_{11}(\tau)$ is obtained from Eq. (3) by taking $s(x)=0$ (i.e. variance of the signal).

In this study, we will use the look-up-table method to obtain $\overline{U_{\perp}}$ from $r_{12}(\tau)$. A look-up table is created with values of the theoretical $r_{12}(\tau)$ (using Eq. 3) given a range of $\overline{U_{\perp}}$ values (resolution of $0.1 \mathrm{~m} \mathrm{~s}^{-1}$ ) and time-lag values (resolution of $0.002 \mathrm{~s}$, equal to the measurement frequency of the scintillometer) (van Dinther and Hartogensis, 2014). Note that $U_{\perp}(x)$ is assumed to be constant when creating the lookup table. The estimate of $\overline{U_{\perp}}$ is obtained by comparing the measured $r_{12}(\tau)$ values to the theoretical $r_{12}(\tau)$ values of the look-up table. The theoretical $r_{12}(\tau)$ that has the best fit with the measured $r_{12}(\tau)$ thus yields the value of $\overline{U_{\perp}}$.

The effects of having a variable $U_{\perp}(x)$ on $r_{12}(\tau)$ and thus on $\overline{U_{\perp}}$ will be investigated by means of four cases (see Sect. 4.3). For these four cases Eq. (3) is integrated over the 136 range gates given the different values for $U_{\perp}(x)$ estimated by the lidar. In this study $r_{12}(\tau)$, and thus $\overline{U_{\perp}}$, are determined over intervals of $10 \mathrm{~s}$. For the comparison between the scintillometer and lidar the $10 \mathrm{~s} \overline{U_{\perp}}$ values are arithmetically averaged to $10 \mathrm{~min}$.

\subsection{Doppler lidar}

In this study, a HALO Photonics (Malvern, UK) Stream Line scanning Doppler heterodyne lidar is used. Full details of this type of lidar are described in Hirsikko et al. (2014) and only briefly summarized here. The lidar emits pulses of radiation at a wavelength of $1.5 \mu \mathrm{m}$; any backscattered radiation from aerosols is used to estimate wind in the atmosphere by assuming that aerosols are perfect tracers of the wind. The pulse repetition rate is $15000 \mathrm{~Hz}$; a $1 \mathrm{~s}$ ray is obtained from the accumulation of 15000 pulses. In the returned signal there is a Doppler shift, which enables calculation of the Doppler velocity, i.e. the velocity component in the direction in which the lidar beam is pointing (also referred to as radial or along-beam wind).

In this study, the crosswind component of the wind speed is needed in order to compare with scintillometer estimates. Note that also a sonic anemometer can yield valuable information about the local wind field above cities. However, in this study the interest is in the variability of $U_{\perp}$ along a path, which can be estimated from the radial Doppler velocities by applying the duo-beam method (Wood et al., 2013c). The method determines the horizontal wind speed and wind direction using trigonometric identities, from which $U_{\perp}(x)$ can be determined.

The duo-beam method relies, as the name implies, on two sets of measurements from the lidar: at two different azimuths (i.e. beam-pointing directions in the horizontal plane). A detailed description of this method is given in Wood et al. (2013c); a brief outline of the method is given here. The radial velocity $\left(V_{\mathrm{b}}^{\mathrm{g}}\right)$ for each range gate $(\mathrm{g})$, as estimated by the lidar, and beam number (b) is given by

$V_{\mathrm{b}}^{\mathrm{g}}=U^{\mathrm{g}} \cos \left(\phi^{\mathrm{g}}+\pi-\theta_{\mathrm{b}}\right)$,

where $U^{\mathrm{g}}$ is the transect wind speed, $\phi^{\mathrm{g}}$ is the wind direction bearing from north, and $\theta_{\mathrm{b}}$ is the bearing of the beam angle. When applying Eq. (4) for two beams, with different 
$\theta_{\mathrm{b}}$, the two unknowns $U^{\mathrm{g}}$ and $\phi^{\mathrm{g}}$ can be solved, by assuming $V_{1}^{\mathrm{g}}=V_{2}^{\mathrm{g}}$. From $U^{\mathrm{g}}$ and $\phi^{\mathrm{g}}$, the value of $U_{\perp}$ can be obtained for each range gate. It is implicit in this method that the wind field is homogeneous between the two lidar beams. Clearly this is not the case in the atmosphere, and one might expect the effects to average out well above buildings (e.g. often assumed so above the roughness sublayer; Roth, 2000; Kastner-Klein and Rotach, 2004). But at heights within, say, 2-3 mean building heights, there will inevitably be error, perhaps including bias, caused by this implicit assumption.

The fixed resolution of the radial wind (of $0.023 \mathrm{~m} \mathrm{~s}^{-1}$ ) also limits the duo-beam method; i.e. in general as the beam separation becomes infinitesimally small, so does the need for accuracy to become infinitesimally fine. Hence, a key drawback of the method is that, when winds are nearly parallel to the path, winds cannot be estimated correctly.

\section{Experimental setup}

The measurements investigated in the present study were taken from 1 to 15 October 2013. The measurement devices used in this study are a scintillometer, a Doppler lidar, and two sonic anemometers. The layout of the measurement devices is given in Fig. 1.

The scintillometer used in this study is a BLS900 (Scintec, Rottenburg, Germany) running with SRun software version 1.09. Note that in this study the output of $\overline{U_{\perp}}$, as given from SRun, is not used. The BLS900 is a scintillometer with two transmitters and one receiver. Raw signal intensities were measured and stored at a frequency of $500 \mathrm{~Hz}$. The setup of the scintillometer is the same as that of other recent Helsinki scintillometer work (Wood et al., 2013b). The scintillometer measured over a path of $4.2 \mathrm{~km}$. The transmitter unit was placed on a roof section of Hotel Torni at a height of $67 \mathrm{~m}$, while the receiver was placed on a roof near the so-called SMEAR-III-Kumpula station at a height of $52.9 \mathrm{~m}$ (see Fig. 1). The surrounding areas have average building heights of 24 and $20 \mathrm{~m}$, and zero-plane displacement heights of 15 and $13 \mathrm{~m}$, at the transmitter and receiver, respectively (Nordbo et al., 2013). The orientation of the scintillometer was nearly north-south $\left(17^{\circ}\right)$; therefore, the wind was nearly perpendicular to the scintillometer path when it was blowing from the east or west. In this study, $U_{\perp}$ is defined as positive when the wind is blowing from the west into the path.

The lidar was placed at a height of $45 \mathrm{~m}$ near the receiver of the scintillometer. Each ray lasts for $1 \mathrm{~s}$ and is repeated every $4 \mathrm{~s}$. The lidar's operational schedule only allowed two azimuth angles for this study (174 and $\left.196^{\circ}\right)$ within each 5 min; see Fig. 1. This pair was wider apart than desired, due to line-of-sight issues. The elevation of the beam was $0.45^{\circ}$. The lidar data are given in a series of $30-\mathrm{m}$ range gates centred at distances $105-9585 \mathrm{~m}$ from the instrument, but data were only needed until $4155 \mathrm{~m}$ (i.e. 136 range gates corresponding to the $4.19 \mathrm{~km}$ length of the scintillometer path).
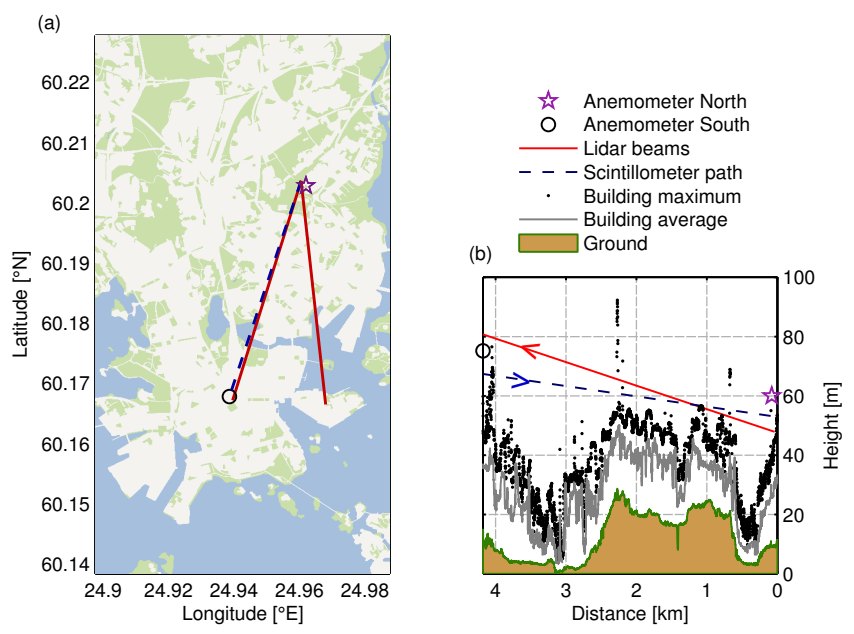

Figure 1. (a) Experimental setup with the locations of the instruments in Helsinki indicated, including Doppler lidarbeam azimuths of 174 and $196^{\circ}$; shading is buildings/roads (white), grass/trees (green), and water (blue) (land cover data source: HSY, SeutuCD); the city centre is roughly the lower half of the map area. (b) A cross section (height $\mathrm{m}$ a.s.l.) of the scintillometer beam and lidar $196^{\circ}$ beam; average building height and maximum building height are with respect to $\pm 250 \mathrm{~m}$ laterally of the $196^{\circ}$ beam (building height data source available at https://sui.csc.fi/applications/paituli/ infra.html).

However - given the atmospheric aerosol loading, sensitivity of the instrument, and integration times - sometimes not enough signal returned from the farthest gates and therefore resulting in a limited range of the data. In order to compare the lidar estimates with $\overline{U_{\perp}}$ estimates of the scintillometer, two of the lidar estimates were averaged. Therefore, $\overline{U_{\perp}}$ estimates of the lidar were available intervals of $10 \mathrm{~min}$.

A 3-D sonic anemometer was located at $75 \mathrm{~m}$ height (near the scintillometer transmitter, denoted here as "Anemometer South") and another at $60 \mathrm{~m}$ (near the receiver, denoted here as "Anemometer North"); see Fig. 1. Due to the mast mounting, the wind directions are more uncertain for 0 $50^{\circ}$ for Anemometer North and $50-185^{\circ}$ for Anemometer South. Fortunately, the wind directions during the measurement period were mainly $210-350^{\circ}$. For more details of the anemometer setup see Järvi et al. (2009) and Nordbo et al. (2013). The value of $U_{\perp}$ measured by each of the anemometers was added to the beginning and the end of the lidarpath estimates, giving a more complete data sample of $U_{\perp}(x)$ along the path. The estimates of $U_{\perp}(x)$ were path-averaged according to the scintillometer path-weighting function given by Wang et al. (1978) for fair comparison with $\overline{U_{\perp}}$ estimated by the scintillometer. In cases of missing $U_{\perp}(x)$ data, the path-weighting factors were scaled to a total of $100 \%$ in order to calculate the estimate of $\overline{U_{\perp}}$ from lidar data. Note that, because of the bell-shaped path-weighting function, the anemometer measurements are barely (only for $2.5 \%$ ) included in the path-weighted $U_{\perp}$ estimates over the path. For 
the comparison between lidar and scintillometer, an arbitrary requirement was that at least $50 \%$ of $U_{\perp}(x)$ of the lidar data were available along the scintillometer path.

\section{Results and discussion}

\subsection{Doppler lidar path-resolved crosswinds}

For the lidar, the urban environment is challenging, since the duo-beam method assumes a homogeneous wind field at each range-gate distance. This assumption will be violated to an unknown degree as the pair of beams diverges. However, lidar is probably the only device which can measure the variability of the wind field along a beam. One other, albeit unrealistic, alternative might be to measure the crosswind along a path using multiple anemometers, but this will be a very challenging setup in the urban environment. However, to ensure the quality of the lidar crosswind estimations, conditions are identified for which the lidar differs from the Anemometer South measurements. We evaluate the difference between $U_{\perp}(x)$ estimated by the lidar and $U_{\perp}$ measured by Anemometer South, to see the impact of the wind direction and building height (see Fig. 2). Note that a perfect agreement between the lidar and anemometer estimates is not expected, since the measurement locations are different. In this paper we only compare Anemometer South to the lidar, but comparing Anemometer North gave similar results (not shown here). The first 10 range gates of $U_{\perp}$ of the lidar compared well with that measured by Anemometer South for the time-period studied, with root-mean-square deviation (RMSD) values of $0.57 \mathrm{~m} \mathrm{~s}^{-1}$. Hirsikko et al. (2014) showed for the same experimental setup, but a different time period, a RMSD of $0.53-0.67 \mathrm{~m} \mathrm{~s}^{-1}$ for the Doppler velocity between lidar and sonic anemometer.

It should be noted that the sign of $U_{\perp}(x)$ is determined by the wind direction estimated by the lidar. When the wind is nearly parallel to the path, a small error in the estimated wind direction can result in an error of the sign of $U_{\perp}(x)$. The wind directions for which the wind is nearly parallel to the path (167-227 and 347-47 $)$ are denoted in light-red shading in the lower figure panel (Fig. 2). It can clearly be seen that there is a substantial difference between lidar and anemometer data for these wind directions, especially when the wind is blowing from 200 to $227^{\circ}$. Even sign changes of the difference are observed. The winds from the $200-227^{\circ}$ directions are also strong $\left(>5 \mathrm{~m} \mathrm{~s}^{-1}\right)$. Therefore, the corresponding $U_{\perp}(x)$ values are still moderate (absolute up to $3 \mathrm{~m} \mathrm{~s}^{-1}$ ) for these wind directions. A small error in the wind direction can therefore result in a sign change of a moderate $U_{\perp}(x)$, which is indeed what we see in Fig. 2. Also for the wind direction $347-46^{\circ}$ there is a clear difference between $U_{\perp}(x)$ of the lidar and $U_{\perp}$ of the anemometer, with differences up to $10 \mathrm{~m} \mathrm{~s}^{-1}$. Whilst we might expect differences above the urban canopy layer, to have such large differences for hun-

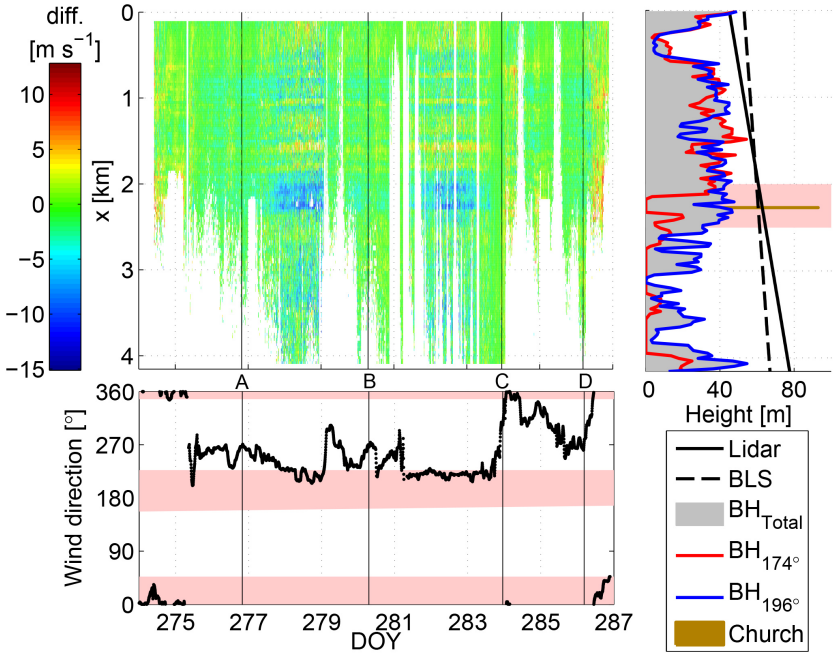

Figure 2. The upper left panel shows the difference in $U_{\perp}$ estimated by the Doppler lidar duo-beam method compared with Anemometer South (colour-bar, Doppler lidar minus sonic anemometer) as a function of lidar beam distance (resolution of $30 \mathrm{~m}$ ) and time (resolution of $10 \mathrm{~min}$, DOY: day of year). A-D are cases (Table 1). The right panel shows the height (ma.s.l.) of the lidar beam and building height $(\mathrm{BH}) \pm 25 \mathrm{~m}$ laterally underneath the paths (total, and under beam with azimuth 174 and $196^{\circ}$ ). When there are no buildings below the path, $\mathrm{BH}$ indicates the height of highest ground point or zero when it is over sea. The lower panel shows wind direction against DOY from Anemometer South.

dreds of metres seems unrealistic. That such large differences in $U_{\perp}(x)$ are unrealistic is also supported by Fig. 3, which shows the estimates of the longitudinal wind component of the lidar along the path. These estimates seem slightly less heterogeneous along the lidar path. Perhaps the larger differences of $U_{\perp}(x)$ are caused by a breakdown of the homogeneity assumption required for the duo-beam method. Whatever the cause, in order to focus on when the method works, it was decided to exclude lidar values for which the wind direction is $167-227$ and $347-46^{\circ}$ for the rest of the study (also when selecting the four cases).

The difference between lidar and anemometer $U_{\perp}$ is also large at 2000-2500 $\mathrm{m}$ along the lidar path (indicated in light red in Fig. 2 on the right). That the lidar estimates of $U_{\perp}(x)$ are unreliable for this part of the path is more clearly visible in Fig. 4, where the average horizontal wind speed $(U)$ and the crosswind speed along the path as estimated by the lidar are shown. Note that in order to make this figure, the nearly parallel wind directions are excluded, as are times when the Doppler lidar data comprised less than $70 \%$ of the total path. The value of $U_{\perp}(x)$ even changes sign at the $2000-2500 \mathrm{~m}$ section along the lidar path. The error in $U_{\perp}(x)$ for this section of the path is probably caused by differences in the wind fields measured by the two beams, since the $196^{\circ}$ beam passes near to a high church tower (Kallio, about $93 \mathrm{~m}$ a.s.1.), which is located $35 \mathrm{~m}$ from the $196^{\circ}$ beam and at $2300 \mathrm{~m}$ 


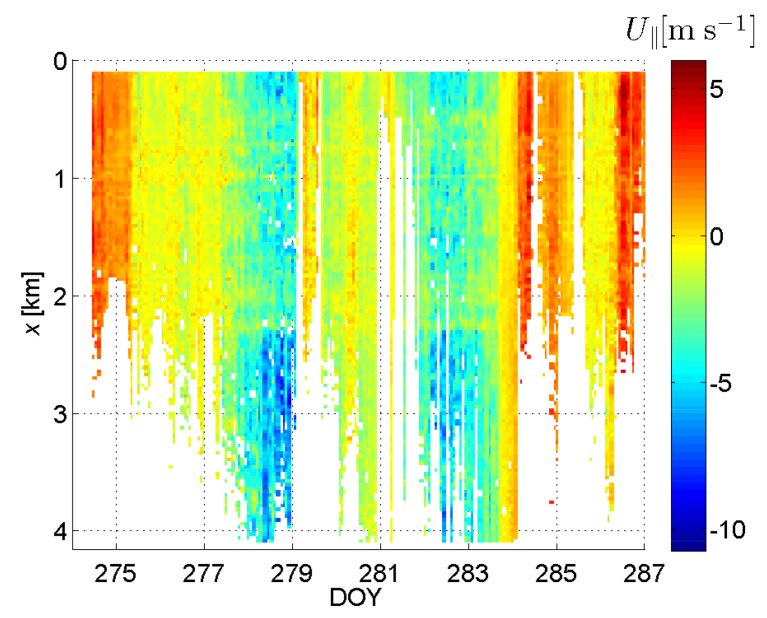

Figure 3. The Doppler wind component as estimated by the Doppler lidar beam of $174^{\circ}$, with lidar beam distance (resolution of $30 \mathrm{~m}$ ) and time (resolution of $10 \mathrm{~min}$ ).
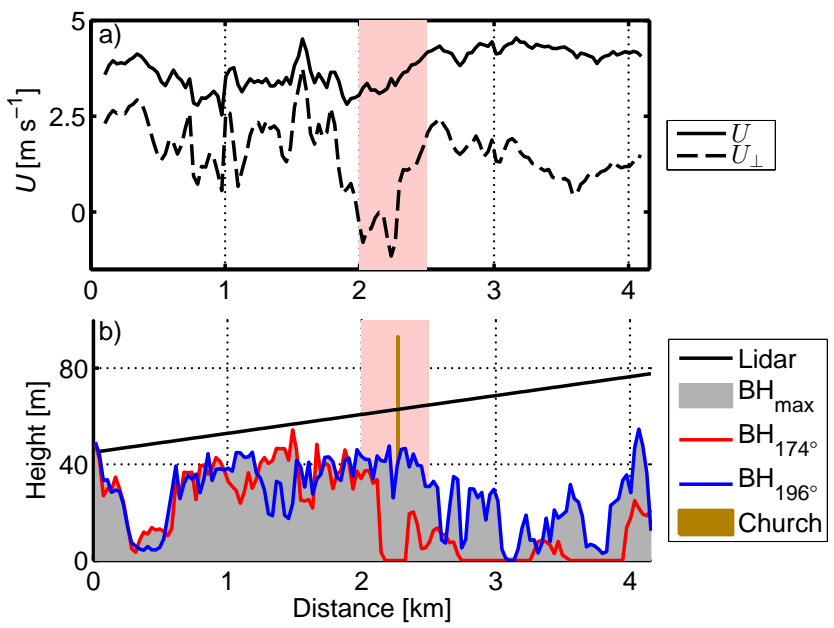

Figure 4. (a) Average horizontal wind speed and crosswind speed estimated by the Doppler lidar. (b) The height (ma.s.l.) of the lidar beam and building height $(\mathrm{BH}) \pm 25 \mathrm{~m}$ laterally underneath the paths (total, and under beam with azimuth 174 and $196^{\circ}$ ). When there are no buildings below the path, $\mathrm{BH}$ indicates the height of highest ground point or zero when it is over sea.

distance from the lidar (see Fig. 1b). Although the church tower is somewhat to the east of the lidar path, it apparently has a significant influence on the wind field estimated by the lidar. The church alters the wind field of one of the lidar paths $\left(196^{\circ}\right)$, while the other beam $\left(174^{\circ}\right)$ does not encounter this alteration. Thus, the wind field sampled by the two lidar beams is not homogeneous, which causes problems for the duo-beam method. Therefore, we also excluded $U_{\perp}(x)$ values estimated by the lidar from 2000 to $2500 \mathrm{~m}$ for the evaluation of scintillometer estimates with lidar estimates. However, in order to evaluate the response of a variable $U_{\perp}(x)$ on $S_{11}(f)$ and $r_{12}(\tau)$, and thus on $\overline{U_{\perp}}$ estimated by the scin-

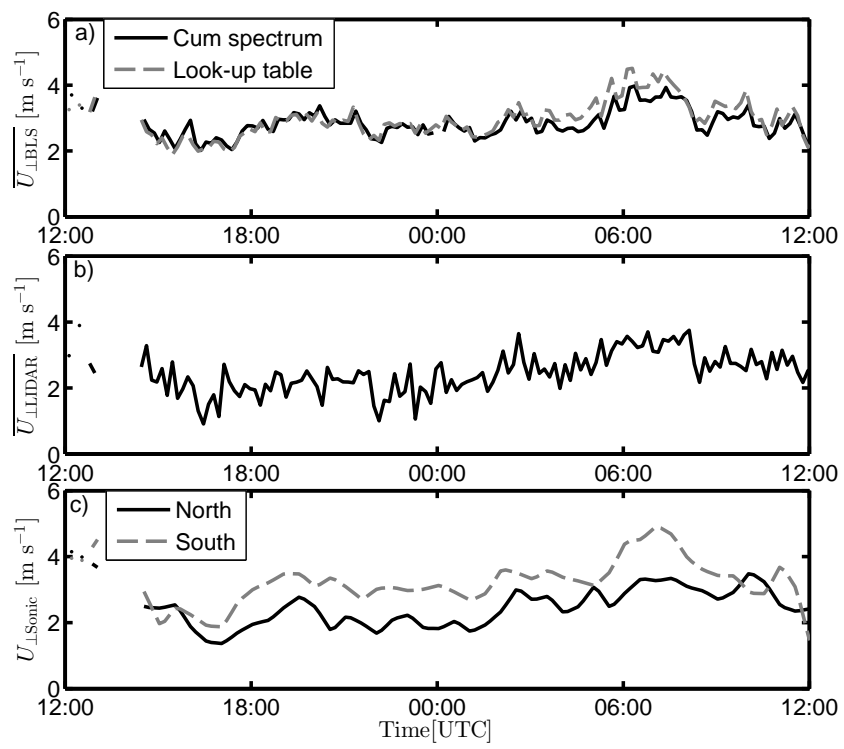

Figure 5. Time series of $U_{\perp}$ as estimated by (a) the scintillometer, (b) the Doppler lidar, and (c) the sonic anemometer for DOY 279 and 280 .

tillometer, the four selected cases need the complete $U_{\perp}(x)$ of the scintillometer path. Therefore, when selecting the four cases the value of $U_{\perp}(x)$ had to be below 1.5. $\overline{U_{\perp}}$ (of the lidar estimates) for $2000 \mathrm{~m} \leq x \leq 2500 \mathrm{~m}$. The four cases selected are indicated in Fig. 2. These cases are spread over the measurement period and have different $\overline{U_{\perp}}$ values. The results of the four cases are presented in Sect. 4.3.

Although the data for which the wind direction was 167227 or $347-46^{\circ}$ are excluded, as are the data at $2000-2500 \mathrm{~m}$ along the lidar path, there are still enough data left for the comparison between lidar and scintillometer. The exclusion resulted in $128810 \mathrm{~min}$ data points (60\% of the data) for the comparison between lidar and scintillometer.

Another issue which can influence the estimate of $U_{\perp}(x)$ of the lidar is temporal variability of $U_{\perp}(x)$. It is worth briefly considering this issue. Temporal variability of $U_{\perp}(x)$ can result in biases and spread in the lidar estimate of $\overline{U_{\perp}}$. In order to investigate the temporal variability of $U_{\perp}$ in these data, the $10 \mathrm{~min}$ variance of $10 \mathrm{~s}$ estimates of $\overline{U_{\perp}}$ made by the look-up-table method is calculated. The variance of $\overline{U_{\perp}}$ was for $86 \%$ of the time below $0.5 \mathrm{~m} \mathrm{~s}^{-1}$ : a moderate temporal variability of $\overline{U_{\perp}}$ in these data.

\subsection{Path-averaged crosswinds}

In this section, $\overline{U_{\perp}}$ obtained from the scintillometer is compared to that estimated from the lidar. Note that the scintillometer path and the lidar duo-beam setup are not sampling the same part of the atmosphere exactly (see Fig. 1). Therefore, a perfect one-to-one correlation cannot be expected. However, the height difference between the scintillometer 


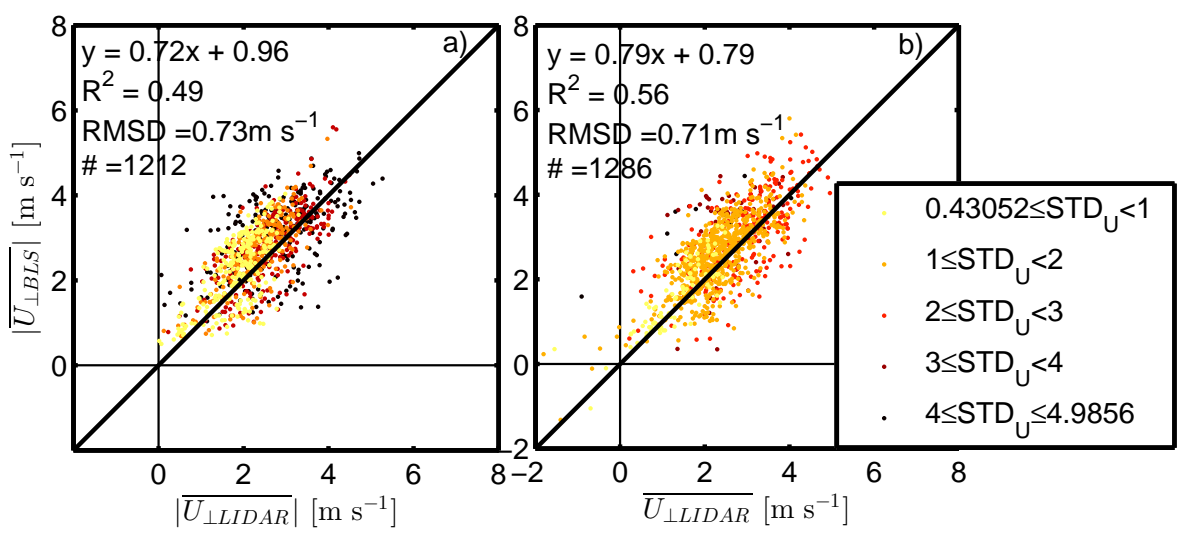

Figure 6. (a) Crosswind $10 \mathrm{~min}$ averages estimated by the scintillometer $\left(\overline{U_{\perp \mathrm{BLS}}}\right)$ using the cumulative-spectrum method against Doppler lidar crosswind $\left(\overline{U_{\perp \text { LIDAR }}}\right)$. (b) Crosswind estimated by the scintillometer using the look-up-table method against lidar data. Both plots are colour-coded with the lidar-derived path-weighted standard deviation of the crosswind along the $4.2 \mathrm{~km}$ path (see legend). The one-to-one lines are shown in thick black.

and the lidar beam causes a negligible difference in the $\overline{U_{\perp}}$ estimates. Assuming a neutral wind profile, the difference in $\overline{U_{\perp}}$ is merely $1.1 \%$ (with the higher $\overline{U_{\perp}}$ estimate at the height of the scintillometer), which suggests that the height difference between the two measurement devices should not influence the comparison. Note that this $1.1 \%$ is only an approximation; in reality the comparison is more complicated since part of the measurements are done just above the urban canopy layer where logarithmic wind profiles are not applicable.

Before looking into detail in the comparison between the lidar and scintillometer estimates of $\overline{U_{\perp}}$, we first show a time series of $U_{\perp}$ as estimated by scintillometer, lidar, and sonic anemometers (Fig. 5). For the scintillometer estimates, it is clear that the cumulative-spectrum method and look-uptable method give very similar results. The lidar estimates of $\overline{U_{\perp}}$ fluctuates more strongly than both the scintillometer and sonic anemometers. However, the lidar does capture the same pattern in $\overline{U_{\perp}}$ as the scintillometer (especially on DOY 280 from 06:00 UTC onwards). For the sonic anemometers it is apparent that they do measure a different value of $U_{\perp}$, which indicates that there is indeed spatial variability of $U_{\perp}$ for this instance.

For the comparison of the lidar and scintillometer we first focus on the result of the cumulative-spectrum method (Fig. 6a). Note that the plots in Fig. 6 are coloured with the standard deviation path-averaged by the scintillometer pathweighting function (standard deviation of $U_{\perp}$; i.e. fluctuations of $U_{\perp}(x)$ in the middle of the path contribute more to $\overline{\mathrm{STD}_{U \perp}}$ than those at the ends of the path). Recall that the sign of $\overline{U_{\perp}}$ is unknown with the cumulative-spectrum method, and thus the absolute values of $\overline{U_{\perp}}$ are compared to each other. The correlation between $\overline{U_{\perp}}$ of the scintillometer and of the lidar gives confidence in the method, with a low RMSD of $0.73 \mathrm{~m} \mathrm{~s}^{-1}$. However, for a higher path- weighted standard deviation along the scintillometer path $\left(\overline{\mathrm{STD}_{U \perp}}\right)$, more scatter occurs between the scintillometer and lidar estimates. Only taking into account the data for which $\overline{\mathrm{STD}_{U \perp}}>2 \mathrm{~m} \mathrm{~s}^{-1}$ leads to an $R^{2}$ value of 0.32 and an RMSD of $0.86 \mathrm{~m} \mathrm{~s}^{-1}$. This higher scatter when $\overline{\mathrm{STD}_{U \perp}}>$ $2 \mathrm{~m} \mathrm{~s}^{-1}$ indicates the difficulty of estimating $\overline{U_{\perp}}$ when the wind field is more variable along the path. An RMSD of $0.73 \mathrm{~m} \mathrm{~s}^{-1}$ is relatively low compared to other studies. For measurements in London (Wood et al., 2013c) for comparable wind conditions, horizontal wind speed RMSDs were found of $0.35 \mathrm{~m} \mathrm{~s}^{-1}$ between two sonic anemometers on the same mast, $0.71-0.73 \mathrm{~m} \mathrm{~s}^{-1}$ between two sonic anemometers on different masts, and $0.65-0.68 \mathrm{~m} \mathrm{~s}^{-1}$ between lidar and sonic anemometers. For $\overline{U_{\perp}}$, Wood et al. (2013c) showed an RMSD of 1.12-2.13 $\mathrm{m} \mathrm{s}^{-1}$ between scintillometer and lidar. For a flat grassland site, where $U_{\perp}(x)$ can be assumed to be rather homogenous, van Dinther et al. (2013) and van Dinther and Hartogensis (2014) showed RMSD values of quality-checked data of $0.41-0.67 \mathrm{~m} \mathrm{~s}^{-1}$ between a scintillometer and sonic anemometer for similar $\overline{U_{\perp}}$ conditions (absolute values are between 0 and $6 \mathrm{~m} \mathrm{~s}^{-1}$ ). Therefore, we can conclude that, despite the higher scatter for variable $U_{\perp}(x)$ conditions, both measurement techniques seem able to obtain an estimate of $\overline{U_{\perp}}$ in this challenging environment.

In Fig. $6 \mathrm{~b}, \overline{U_{\perp}}$ obtained by the look-up-table method is compared to the lidar estimates. Note that the following regression statistics are obtained when absolute $\overline{U_{\perp}}$ values are considered: RMSD of $0.73 \mathrm{~m} \mathrm{~s}^{-1}, y=0.76 x+0.83$, and $R^{2}=0.53$. Just like the cumulative-spectrum method, there is a clear correlation between $\overline{U_{\perp}}$ estimated by the scintillometer and that estimated by the lidar. The regression statistics of the absolute $\overline{U_{\perp}}$ are similar with the same RMSD and similar regression equation (although a slightly better fit for the look-up-table method). The scatter of $\overline{U_{\perp}}$ of the look-uptable method with the lidar estimates is somewhat lower than 
Table 1. Crosswind for the four cases estimated by the Doppler lidar and scintillometer (using either cumulative spectra, CS, or time-lagged correlation function, $\left.r_{12}(\tau)\right) . \overline{U_{\perp} \text { var } U \perp}$ is given by the theoretical CS and $r_{12}(\tau)$ using the variable $U_{\perp}(x)$ estimated by the lidar.

\begin{tabular}{ccc|cc|cc|cc}
\hline Case & DOY & HH:MM & \multicolumn{2}{|c|}{ Lidar } & \multicolumn{2}{c|}{ CS } & \multicolumn{2}{c}{$r_{12}(\tau)$} \\
\cline { 4 - 8 } & & (UTC) & $\overline{U_{\perp}}$ & $\overline{\text { STD }_{U \perp *}}$ & $\overline{U_{\text {BLS }}}$ & $\overline{U_{\text {var} U \perp}}$ & $\overline{U_{\text {BLS }}}$ & $\overline{U_{\text {var } U \perp}}$ \\
\hline A & 276 & $19: 47$ & 2.8 & 0.36 & 3.5 & 2.6 & 3.2 & 2.5 \\
B & 280 & $06: 57$ & 3.3 & 0.39 & 3.4 & 3.1 & 4.1 & 3.0 \\
C & 283 & $22: 57$ & 1.6 & 0.63 & 1.6 & 1.4 & 0.8 & 0.8 \\
D & 286 & $04: 27$ & 3.9 & 0.41 & 3.5 & 3.9 & 3.1 & 3.1 \\
\hline
\end{tabular}

that of the cumulative-spectrum method with an $R^{2}$ value of 0.53 compared to 0.47 . For the look-up table, the scatter is also higher $\left(R^{2}\right.$ of 0.37 and RMSD of $\left.0.88 \mathrm{~m} \mathrm{~s}^{-1}\right)$ when $U_{\perp}(x)$ is variable $\left(\overline{\operatorname{STD}}_{U \perp}>2 \mathrm{~m} \mathrm{~s}^{-1}\right)$.

Overall, both scintillometer methods are able to obtain a similar $\overline{U_{\perp}}$ estimate as that of the lidar. This indicates that both the lidar and scintillometer offer the potential to obtain an estimate of $\overline{U_{\perp}}$ over the complex urban environment. However, remember that, in order to achieve these results, certain wind directions and a certain section of the path were not taken into account (see Sect. 4.1). The look-up-table method showed the best results, with the lowest RMSD and scatter.

\subsection{Variable crosswinds along the path}

Four cases were selected to investigate the influence of a variable $U_{\perp}(x)$ on $S_{11}(f)$ and $r_{12}(\tau)$ : A, B, C, and D (see top panels in Fig. 7 and Table 1). As a measure of the variability of $U_{\perp}(x)$, the weight-averaged standard deviation of $U_{\perp}(x)$ is normalized by $\overline{U_{\perp}}\left(\overline{\operatorname{STD}}_{U \perp *}\right)$. For the four cases, the theoretical $S_{11}(f)$ and $r_{12}(\tau)$ are calculated using Eqs. (1) and (3), respectively.

We first focus on the cumulative scintillation spectra (CS, given in the middle panels of Fig. 7). Remember that the cumulative-spectrum method determines $\overline{U_{\perp}}$ from the frequencies for which the CS is $0.5,0.6,0.7,0.8$, and 0.9 . The spectra in Fig. 7 are zoomed such that the relevant points for this method stand out. For simplicity we abbreviate the cumulative spectrum obtained from the scintillometer as $\mathrm{CS}_{\mathrm{BLS}}$, the cumulative spectrum obtained from Eq. (1) using $U_{\perp}(x)$ of the lidar as $\mathrm{CS}_{\mathrm{var} U \perp \text {, and the cumulative spectrum }}$ obtained from Eq. (1) using $\overline{U_{\perp}}$ of the lidar as $\mathrm{CS}_{\text {const } U \perp \text {. }}$

There is a difference between $\mathrm{CS}_{\mathrm{var} U \perp}$ and $\mathrm{CS}_{\text {const } U \perp}$ for all four cases. Therefore, the CS is indeed influenced by a variable $U_{\perp}(x)$ as was suggested by van Dinther et al. (2013). Recall that, when a CS point shifts to a higher frequency, the retrieved value of $\overline{U_{\perp}}$ will be higher, and the other way around (see Eq. 2). The CS points of 0.5, 0.6, and 0.7 lie at lower frequencies for $\mathrm{CS}_{\mathrm{var} U \perp}$ than for $\mathrm{CS}_{\text {const } U \perp \text {, while }}$ the $0.9 \mathrm{CS}$ point lies at higher frequencies. $\mathrm{CS}_{\mathrm{BLS}}$ is more similar to $\mathrm{CS}_{\mathrm{var} U \perp}$ than to $\mathrm{CS}_{\text {const } U \perp \text {, which indicates that }}$ Eq. (1) is also applicable when $U_{\perp}(x)$ is variable.
The results of applying the cumulative-spectrum method to $\mathrm{CS}_{\mathrm{BLS}}$ and $\mathrm{CS}_{\mathrm{var} U \perp}$ are given in Table 1. If the assumption of the cumulative-spectrum methods - that $C_{\mathrm{CS}}$ of Eq. (2) is constant - also holds for variable $U_{\perp}(x)$, then the value of $\overline{U_{\perp}}$ of the lidar should be identical to that of $\overline{U_{\mathrm{CS}_{\text {var } U \perp}}}$. For case $\mathrm{D}$ this is indeed true.

However, for case A, B, and $\mathrm{C}, \overline{U_{\mathrm{CS}_{\mathrm{var}} U \perp}}$ is $0.2 \mathrm{~m} \mathrm{~s}^{-1}$ lower than $\overline{U_{\mathrm{LiIDAR}}}$. Therefore, the assumption that $C_{\mathrm{CS}}$ is constant does not hold. However, the error that is made in $\overline{U_{\perp}}$ is small $\left(0.2 \mathrm{~m} \mathrm{~s}^{-1}\right)$, which is due to the cumulativespectrum method calculating $\overline{U_{\perp}}$ for five frequency points and then averaging these to obtain one value for $\overline{U_{\perp}}$ (see Sect. 2.1.1). For the $0.5,0.6$, and $0.7 \mathrm{CS}$ points, $\overline{U_{\mathrm{CS}_{\mathrm{var}} U \perp}}$ is underestimated; while for the $0.9 \mathrm{CS}$ point, $\overline{U_{\mathrm{CS}_{\mathrm{var}} U \perp}}$ is overestimated. Therefore, applying a method with only one frequency point to obtain $\overline{U_{\perp}}$ is more likely to have a higher error. This makes the cumulative-spectrum method the most suitable method for obtaining $\overline{U_{\perp}}$ from $S_{11}(f)$ when $U_{\perp}(x)$ is variable, compared to other methods suggested by van Dinther et al. (2013). Alternatively, to obtain $\overline{U_{\perp}}$ even more reliably from $S_{11}(f)$ in variable $U_{\perp}(x)$ conditions, an approach similar to the look-up-table method can be applied. A look-up table can be created of the theoretical CS for different $\overline{U_{\perp}}$ values and also different variabilities of $U_{\perp}(x)$.

Next we focus on the results of the look-up-table method, which relies on $r_{12}(\tau)$ to obtain $\overline{U_{\perp}}$ (given in the bottom panels of Fig. 7). For all cases except case B, there is a substantial difference in magnitude between $r_{12 \mathrm{var}} U \perp(\tau)$ (grey solid lines) and $r_{12 \text { const } U \perp}(\tau)$ (grey dotted lines). However, the magnitude of $r_{12}(\tau)$ does not influence $\overline{U_{\perp}}$ obtained by the look-up-table method, but the shape of $r_{12}(\tau)$ does. The shape of $r_{12}(\tau)$ also changes when $U_{\perp}(x)$ is variable: it becomes wider. For cases $\mathrm{C}$ and $\mathrm{D}, r_{12 \operatorname{var}} U \perp(\tau)$ resembles $r_{12 \mathrm{BLS}}(\tau)$ clearly better than $r_{12 \text { const } U \perp}(\tau)$. This resemblance indicates that the theoretical model of Lawrence et al. (1972) (Eq. 3) can be used to obtain $r_{12}(\tau)$ also given a variable $U_{\perp}(x)$. The fact that variable $U_{\perp}(x)$ causes a wider $r_{12}(\tau)$ can cause an underestimation of $\overline{U_{\perp}}$ obtained by the scintillometer, since a wider $r_{12}(\tau)$ is normally associated with lower $\overline{U_{\perp}}$ values. For the four cases selected in this study $\overline{U_{\perp}}$ calculated from $r_{12 \text { var } U \perp}$ is indeed lower than $\overline{U_{\perp}}$ estimated by the lidar (see Table 1). The error is here defined as the difference between $\overline{U_{\perp}}$ estimated by the lidar and $\overline{U_{\perp}}$ 

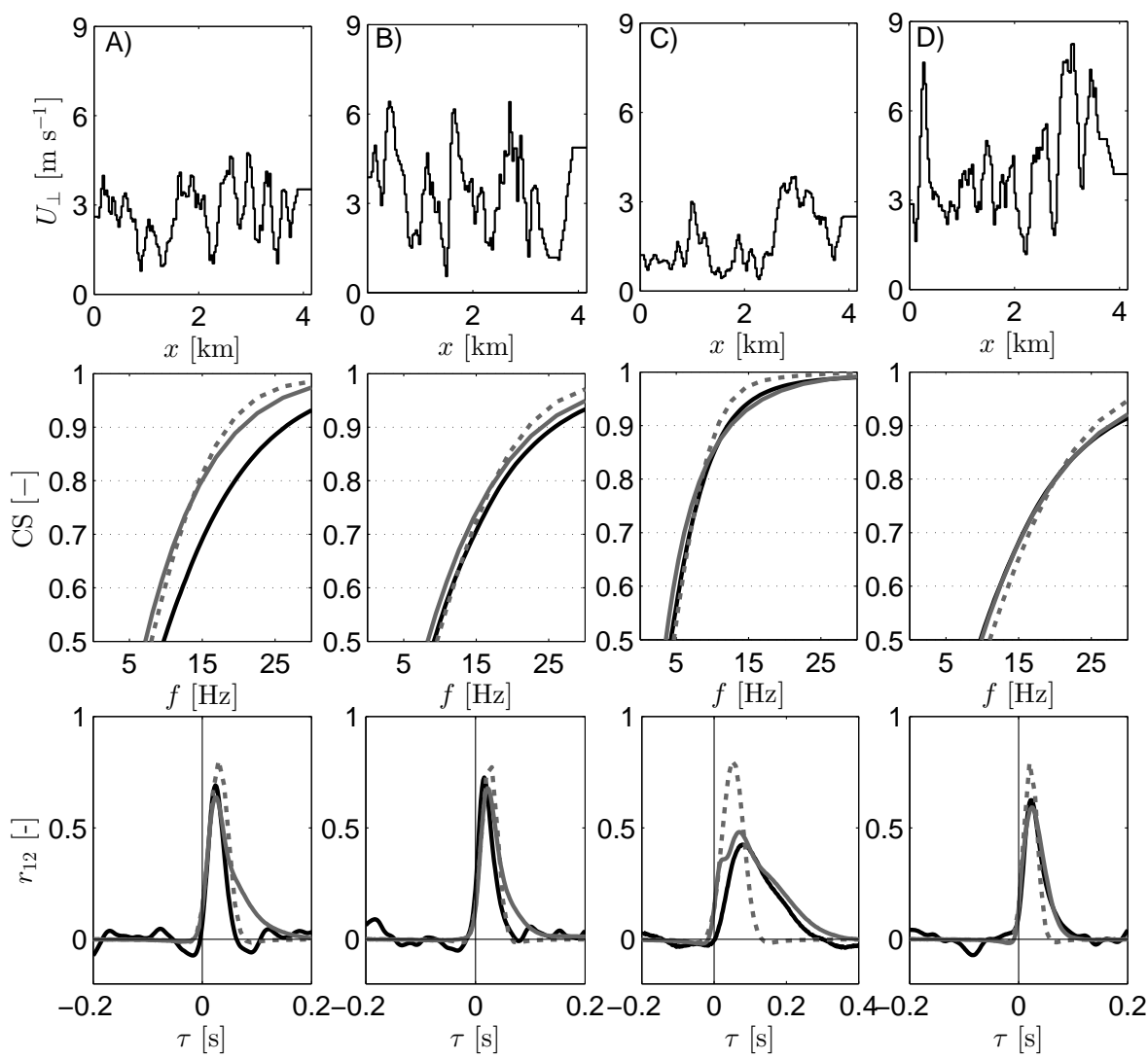

Figure 7. Four cases (A, B, C, and D): in the top panels the transect of $U_{\perp}(x)$, in the middle panels the corresponding CS, and in the lower panels the corresponding $r_{12}(\tau)$. The estimated CS and $r_{12}(\tau)$ of the scintillometer are given as black solid lines, the theoretical CS and $r_{12}(\tau)$ given $U_{\perp}(x)$ of the Doppler lidar are given as solid grey lines, and the theoretical CS and $r_{12}(\tau)$ given $U_{\perp}(x)=\overline{U_{\perp}}$ are given as dashed grey lines.

obtained from $r_{12}(\tau)$. For case $\mathrm{C}$ and $\mathrm{D}$, the error is high with a value of $0.8 \mathrm{~m} \mathrm{~s}^{-1}$. This high error is caused by the fact that for these two cases not only is $r_{12}(\tau)$ lowered by the variable $U_{\perp}(x)$, but the peak in $r_{12}(\tau)$ also changes location and $r_{12}(\tau)$ becomes much wider due to the variable $U_{\perp}(x)$. For these cases $\overline{\mathrm{STD}_{U \perp *}}$ is also high with values of 0.63 and 0.41 , respectively. Although the error with the lidar estimates is high for case $\mathrm{C}$ and $\mathrm{D}$, the estimated $\overline{U_{\perp \mathrm{BLS}}}$ of the look-uptable method are identical to that of $r_{12 \operatorname{var}} U \perp(\tau)$. Therefore, if the look-up table were expanded to also include a variable $U_{\perp}(x)$ field, the results of the look-up-table method in a more challenging environment could be improved. The underestimation of $\overline{U_{\perp}}$ given in the cases is however not clearly visible in the comparison of lidar and scintillometer (see Sect. 4.2, Fig. 6). However, we do see that a higher $\overline{\mathrm{STD}_{U \perp}}$ causes more scatter between $\overline{U_{\perp}}$ of the scintillometer and lidar.

From the analysis of these four cases, it follows that the present cumulative-spectrum method is better equipped to obtain $\overline{U_{\perp}}$ than the look-up-table method. However, the lookup-table method can be adjusted to take into account the variability of $U_{\perp}(x)$. The underestimation of $\overline{U_{\perp}}$ found for the four cases for both methods was not clearly distinguishable in Sect. 4.2, though more scatter occurred between $\overline{U_{\perp}}$ estimated by scintillometer and lidar when $\overline{\mathrm{STD}_{U \perp}}$ was high $\left(>2 \mathrm{~m} \mathrm{~s}^{-1}\right)$.

\section{Conclusions and outlook}

In this study, estimates of $U_{\perp}$ above the urban environment of Helsinki from sonic anemometers and Doppler lidar data were compared with scintillometer data. The anemometers measured at either ends of the scintillometer path, and the lidar was measuring alongside the scintillometer path. For the lidar duo-beam method, sign problems of $U_{\perp}$ naturally occurred when the wind direction was parallel to the scintillometer path (167-227 and 347-47 ). In the middle of the path (at 2000-2500 m) a church tower near one of the lidar beams resulted in problems, presumably because of the heterogeneity it introduced in the wind field. Therefore, for the comparison with the scintillometer these points were excluded.

For the scintillometer, two different methods were tested: the cumulative-spectrum method (van Dinther et al., 2013), 
based on $S_{11}(f)$, and the look-up-table method (van Dinther and Hartogensis, 2014), based on $r_{12}(\tau)$. Both methods gave similar results to the lidar estimates, albeit with scatter between the lidar and the scintillometer (especially for conditions for which $\overline{\mathrm{STD}_{U \perp}}>2 \mathrm{~m} \mathrm{~s}^{-1}$ ). Still, given that the lidar and scintillometer did not sample the exact same area in this urban environment, the good fit and low RMSD $(\leq$ $0.73 \mathrm{~m} \mathrm{~s}^{-1}$ ) indicate that both measurement devices are able to obtain $\overline{U_{\perp}}$ estimates, given the data treatment applied in this study. For the scintillometer the method relying on $r_{12}(\tau)$ (look-up-table method) is preferable, since $r_{12}(\tau)$ is determinable over a short timescale $(\sim 10 \mathrm{~s})$ compared to scintillation spectra $(\sim 10 \mathrm{~min})$, and it also includes information about the sign of $\overline{U_{\perp}}$.

Four cases were selected to investigate the influence of a variable $U_{\perp}(x)$ on $\overline{U_{\perp}}$ estimated by the scintillometer. Variability of $U_{\perp}(x)$ causes only a slight difference between $\overline{U_{\perp}}$ estimated by the cumulative-spectrum method and lidar (error $\leq 0.2 \mathrm{~m} \mathrm{~s}^{-1}$ ). $r_{12}(\tau)$ was more affected by a variable $U_{\perp}(x)$ field than $S_{11}(f)$, leading to higher errors in $\overline{U_{\perp}}$ obtained by the look-up-table method (error $\leq 0.8 \mathrm{~m} \mathrm{~s}^{-1}$ ). The look-up-table method can however be adjusted to include heterogeneous wind fields, thereby probably making the scintillometer more suitable to estimate $\overline{U_{\perp}}$ in a more challenging environment.

In this study the focus was on the influence of spatial variability of $U_{\perp}(x)$ on scintillometer $\overline{U_{\perp}}$ estimates. However, temporal variability of $U_{\perp}(x)$ will also influence the estimates of $\overline{U_{\perp}}$. We expect that this temporal variability has the same influence as the spatial variability: a smoothing of $S_{11}(f)$ and a widening of $r_{12}(\tau)$. However, methods that rely on $r_{12}(\tau)$ are likely not affected by temporal variability of $U_{\perp}(x)$, since $r_{12}(\tau)$ is determined over a reasonably short time interval $(\sim 10 \mathrm{~s})$. Methods that rely on $S_{11}(f)$ are more likely to be affected by a temporal variability of $U_{\perp}(x)$, since $S_{11}(f)$ is determined over a relatively long time inter$\operatorname{val}(\sim 10 \mathrm{~min})$.

In the future, by applying two scintillometers with paths perpendicular to each other, not only $\overline{U_{\perp}}$ but also the wind direction and horizontal wind speed could be estimated (Andreas, 2000), thereby obtaining an area-averaged value of the horizontal wind speed and wind direction above an urban environment. Compared to a Doppler lidar the scintillometer is less expensive and easier to use. A path-averaged value of wind direction and horizontal wind speed would be directly useful for nowcasting for meteorology and for atmospheric composition (AC), and also in the development of models of $\mathrm{AC}$ and numerical weather prediction.

Acknowledgements. The authors would like to thank Leena Järvi, Rostislav Kouznetsov, Anne Hirsikko, and Ville Vakari for their help with instrumental setup and preliminary data analysis and Kari Riikonen, Erkki Siivola, Petri Keronen, and Sami Haapanala for their technical support. D. van Dinther and O. K. Hartogensis were supported by the Knowledge for Climate project Theme 6 en- titled "High Quality Climate Projections" (KVK-HS2). C. R. Wood was supported by the EC FP7 ERC grant no. 227915, "Atmospheric planetary boundary layers: Physics, modelling and role in Earth system". We would also like to thank the anonymous reviewers for their valuable comments, which helped to improve the quality of the manuscript.

Edited by: G. Baumgarten

\section{References}

Andreas, E. L.: Obtaining Surface Momentum and Sensible Heat Fluxes from Crosswind Scintillometers, J. Atmos. Ocean. Technol., 17, 3-16, 2000.

Bornstein, R. D. and Johnson, D. S.: Urban-rural wind velocity differences, Atmos. Environ., 11, 597-604, 1977.

Briggs, B. H., Phillips, G. J., and Shinn, D. H.: The Analysis of Observations on Spaced Receivers of the Fading of Radio Signals, Proc. Phys. Soc. B, 63, 106-121, 1950.

Clifford, S. F.: Temporal-frequency spectra for a spherical wave propagating through atmospheric turbulence, J. Opt. Soc. Am., 61, 1285-1292, 1971.

De Bruin, H.: Introduction: renaissance of scintillometry, Bound.Lay. Meteorol. 105, 1-4, 2002.

Hirsikko, A., O'Connor, E. J., Komppula, M., Korhonen, K., Pfüller, A., Giannakaki, E., Wood, C. R., Bauer-Pfundstein, M., Poikonen, A., Karppinen, T., Lonka, H., Kurri, M., Heinonen, J., Moisseev, D., Asmi, E., Aaltonen, V., Nordbo, A., Rodriguez, E., Lihavainen, H., Laaksonen, A., Lehtinen, K. E. J., Laurila, T., Petäjä, T., Kulmala, M., and Viisanen, Y.: Observing wind, aerosol particles, cloud and precipitation: Finland's new groundbased remote-sensing network, Atmos. Meas. Tech., 7, 13511375, doi:10.5194/amt-7-1351-2014, 2014.

Järvi, L., Hannuniemi, H., Hussein, T., Junninen, H., Aalto, P., Hillamo, R., Mäkelä, T., Keronen, P., Siivola, E., Vesala, T., and Kulmala, M.: The urban measurement station SMEAR III: Continuous monitoring of air pollution and surface-atmosphere interactions in Helsinki, Finland, Boreal Environ. Res., 14, 86-109, 2009.

Kastner-Klein, P. and Rotach, M. W.: Mean flow and turbulence characteristics in an urban roughenss sublayer, Bound.-Lay. Meteorol., 111, 58-84, 2004.

Kolmogorov, A. N.: The local structure of turbulence in an incompressible viscous fluid for very large Reynolds numbers, Dokl. Akad. Nauk. SSSR+, 30, 299-303, 1941.

Lawrence, R. S., Ochs, G. R., and Clifford, S. F.: Use of scintillations to measure average wind across a light beam., Appl. Opt., 11, 239-43, 1972.

Meijninger, W. M. L., Green, A. E., Hartogensis, O. K., Kohsiek, W., Hoedjes, J. C. B., Zuurbier, R. M., and De Bruin, H. A. R.: Determination of area-averaged water vapour fluxes with large aperture and radio wave scintillometers over a heterogeneous surface - Flevoland Field Experiment, Bound.-Lay. Meteorol., 105, 63-83, 2002a.

Meijninger, W. M. L., Hartogensis, O. K., Kohsiek, W., Hoedjes, J. C. B., Zuurbier, R. M., and De Bruin, H. A. R.: Determination of area-averaged sensible heat fluxes with a large aperture 
scintillometer over a heterogeneous surface - Flevoland field experiment, Bound.-Lay. Meteorol., 105, 37-62, 2002 b.

Nieveen, J. P., Green, A. E., and Kohsiek, W.: Using a large-aperture scintillometer to measure absorption and refractive index fluctuations, Bound.-Lay. Meteorol., 87, 101-116, 1998.

Nordbo, A., Järvi, L., Haapanala, S., Moilanen, J., and Vesala, T.: Intra-City Variation in Urban Morphology and Turbulence Structure in Helsinki, Finland, Bound.-Lay. Meteorol., 146, 469-496, 2013.

Poggio, L. P., Furger, M., Prévôt, A. H., Graber, W. K., and Andreas, E. L.: Scintillometer Wind Measurements over Complex Terrain, J. Atmos. Ocean. Technol., 17, 17-26, 2000.

Roth, M.: Review of atmospheric turbulence over cities, Q. J. Roy. Meteor. Soc., 126, 941-990, 2000.

van Dinther, D. and Hartogensis, O. K.: Using the Time-LagCorrelation function of Dual-Aperture-Scintillometer measurements to obtain the Crosswind, J. Atmos. Ocean. Technol., 31, 62-78, 2014.

van Dinther, D., Hartogensis, O. K., and Moene, A. F.: Crosswinds from a Single-Aperture Scintillometer Using Spectral Techniques, J. Atmos. Ocean. Technol., 30, 3-21, 2013.

Wang, T., Ochs, G. R., and Clifford, S. F.: A saturation-resistant optical scintillometer to measure $C_{n}^{2}$, J. Opt. Soc. Am., 68, 334338,1978 .
Wang, T. I., Ochs, G. R., and Lawrence, R. S.: Wind measurements by the temporal cross-correlation of the optical scintillations, Appl. Opt., 20, 4073-81, 1981.

Ward, H. C., Evans, J. G., and Grimmond, C. S. B.: Effects of Non-Uniform Crosswind Fields on Scintillometry Measurements, Bound.-Lay. Meteorol., 141, 143-163, 2011.

Wood, C. R., Järvi, L., Kouznetsov, R. D., Nordbo, A., Joffre, S., Drebs, A., Vihma, T., Hirsikko, A., Suomi, I., Fortelius, C., O’Connor, E., Moiseev, D., Haapanala, S., Moilanen, J., Kangas, M., Karppinen, A., Vesala, T., and Kukkonen, J.: An Overview of the Urban Boundary Layer Atmosphere Network in Helsinki, B. Am. Meteorol. Soc., 94, 1675-1690, 2013a.

Wood, C. R., Kouznetsov, R. D., Gierens, R., Nordbo, A., Järvi, L., Kallistratova, M. A., and Kukkonen, J.: On the Temperature Structure Parameter and Sensible Heat Flux over Helsinki from Sonic Anemometry and Scintillometry., J. Atmos. Ocean. Technol. 14, 1604-1615, 2013b.

Wood, C. R., Pauscher, L., Ward, H. C., Kotthaus, S., Barlow, J. F., Gouvea, M., Lane, S. E., and Grimmond, C. S. B.: Wind observations above an urban river using a new lidar technique, scintillometry and anemometry., Sci. Total Environ., 442, 527-33, 2013c. 